\title{
Comparison of TOF MRA, Contrast-Enhanced MRA and Subtracted CTA from CTP in Residue Evaluation of Treated Intracranial Aneurysms
}

\author{
Tolga Turan DUNDAR ${ }^{1}$, Ayse ARALASMAK ${ }^{2}$, Huseyin OZDEMIR ${ }^{2}$, Mehmet Hakan SEYITHANOGLU ${ }^{1}$, \\ Omer UYSAL ${ }^{3}$, Huseyin TOPRAK ${ }^{2}$, Serkan KITIS ${ }^{1}$, Erdinc OZEK ${ }^{1}$, Alpay ALKAN² \\ ${ }^{1}$ Bezmialem Vakif University, Faculty of Medicine, Department of Neurosurgery, Istanbul, Turkey \\ ${ }^{2}$ Bezmialem Vakif University, Faculty of Medicine, Department of Radiology, Istanbul, Turkey \\ ${ }^{3}$ Bezmialem Vakif University, Faculty of Medicine, Department of Biostatistics, Istanbul, Turkey \\ This study has been presented at the $16^{\text {th }}$ World Congress of Neurosurgery in Istanbul, Turkey, between 20 and 25 August, 2017.
}

\section{ABSTRACT}

AIM: To compare effectiveness of contrast-enhanced magnetic resonance angiography (CE-MRA), 3D-Time-of-flight magnetic resonance angiography (3D-TOF-MRA) and subtracted computed tomography angiography from computed tomography perfusion (sub-CTA) in residue evaluation of intracranial aneurysms treated either with coiling or clipping.

MATERIAL and METHODS: Sixteen treated aneurysms, which were evaluated with three methods within two weeks after the operation, were enrolled. The success of each imaging technique in the demonstration of residue aneurysm and nearby vessels was compared by Fisher's Exact Test. The differences among the three methods were evaluated by Cochran's $Q$ test ( $p \leq 0.05)$.

RESULTS: Perfusion abnormality was noted in $81 \%$ of clipped and none of coiled patients. Vessel visualization in the vicinity of aneurysm was better in sub-CTA, followed by CE-MRA. In clipped aneurysms, sub-CTA revealed residue aneurysms in $16.7 \%$ of the patients while 3D-TOF-MRA and CE-MRA revealed none. In coiled aneurysms, CE-MRA revealed residue aneurysms in 100\%, and TOF-MRA in $33.3 \%$ while sub-CTA revealed none. Although dramatic differences were noted in the evaluation of residue aneurysm as well as nearby vessel visualization, no statistical significance was noted due to very few patients in the subcategories. CONCLUSION: This is the first study comparing the effectiveness of CE-MRA, 3D-TOF MRA and sub-CTA in residue aneurysm evaluation although our results were suggestive, not conclusive. Vessel visualization in the vicinity of the aneurysm was better in sub-CTA in all cases regardless of coiling or clipping. Residue aneurysms were more commonly revealed by CE-MRA in coiled patients and more commonly and better shown by sub-CTA in clipped patients in addition to showing perfusion abnormality that was more common in clipped patients.

KEYWORDS: Aneurysm, CE-MRA, TOF-MRA, CTA, CTP, Intracranial

ABBREVIATIONS: CE-MRA: Contrast-enhanced magnetic resonance angiography, CTA: Computed tomography angiography, CTP: Computed tomography perfusion, MRA: Magnetic resonance angiography, 3D-TOF-MRA: Three dimensional time-offlight magnetic resonance angiography, Sub-CTA: Subtracted computed tomography angiography from computed tomography perfusion, MCA: Middle cerebral artery, ICA: Internal carotid artery, CT: Computed tomography, MRI: Magnetic resonance imaging. 


\section{INTRODUCTION}

A ssessment of treated aneurysms is critical and needs effective method to reveal any residue. However, this assessment is speculative as to how long, how often and by which technique the treated aneurysms need to be followed and which subgroups carry a higher or lower risk for reopening. This study compared contrast-enhanced magnetic resonance angiography (CE-MRA), three dimensional timeof-flight magnetic resonance angiography (3D-TOF-MRA) and subtracted computed tomography angiography from computed tomography perfusion (sub-CTA) for the follow-up of treated intracranial aneurysms either with coils or clip.

Intracranial aneurysms are treated either with microneurosurgical clipping or endovascular procedures, e.g. coiling $(13,18)$. Intraarterial digital subtraction angiography (DSA) is considered to be the gold standard for the detection and treatment following of intracranial aneurysms, but this technique is invasive, irradiating and associated with a risk of thromboembolic complications as well as complications related to contrast media injection (14). In many institutions, magnetic resonance angiography (MRA) and computed tomography angiography (CTA) are usually preferred in the follow-up of treated cerebral aneurysms due to their non-invasiveness. However, on control images, these non-invasive imaging techniques become much more challenging in the vicinity of the treated aneurysm owing to the interaction of the various therapeutic devices (i.e., aneurysm clips, embolic coils, and/or stents) with the proton relaxation signal intensity of magnetic resonance imaging (MRI) or photon flux of computed tomography (CT). Coil- or clip-related artifacts hinder the evaluation of possible residue aneurysm as well as vessels in the region of the treated aneurysm. Therefore some institutions still prefer using standard catheter based DSA for evaluation despite its invasiveness (10). In some, DSA is still the primary imaging modality in residue evaluation in the 3 months following clipping of aneurysms in patients with subarachnoid bleeding.

In patients with aneurysmal subarachnoid bleeding, early detection of vasospasm is critical for the treatment and survival of patients. Although DSA is invasive, it is still preferred since it allows intraarterial treatment during imaging of vasospasm. Nowadays, non-invasive imaging to visualize vasospasm and the associated perfusion deficit has become possible via CT perfusion (CTP) $(2,15)$. In new scanners, CTA views can be created from CTP data by subtraction via post-processing. Both perfusion deficit and vasospasm can be evaluated only by CTP imaging without increasing radiation dose. In the literature, TOF-MRA is suggested for residue imaging in aneurysms treated by coiling. TOF-MRA is non-invasive imaging with no need for contrast agent for visualization of brain vessels. Contrast enhanced MRA (CEMRA) is not routinely used for brain vessel imaging. However, it shows brain vessels much better than TOF-MRA because of intravenous contrast injection. Some institutions prefer CEMRA for residue evaluation in aneurysms treated by coiling (17). In our institution, we prefer CE-MRA to TOF-MRA in routine imaging if we use contrast agent for another reason.

In our institution, TOF-MRA, CE-MRA, CTP and DSA can be done for residue evaluations of treated aneurysms.
We retrospectively collected the patients that had undergone TOF-MRA, CE-MRA or CTP within two weeks for residual aneurysm evaluations following treatment either by coiling or clipping, or for another reason such as vasospasm and perfusion deficit. We compared the three imaging modalities in terms of residual aneurysm evaluation.

\section{MATERIAL and METHODS}

All patients harboring ruptured or unruptured intracranial aneurysms were treated by our endovascular team with microneurosurgical clipping or endovascular coiling procedures at our center. Our endovascular team includes neurosurgeons, radiologists, neurologists and anesthesiologists. All patients' examinations were performed by a neurosurgeon (TTD) with 10 years of experience and radiological evaluation was performed by a fellowship educated neuroradiologist (AA) and an interventional neuroradiologist $(\mathrm{HO})$, both with 20 years of experience. They reviewed the cases together and reached a consensus in any disputed case. Approval of the ethical review board of the hospital was obtained for this retrospective study. All procedures performed in studies involving human participants were in accordance with the ethical standards of the institutional and/or national research committee and with the 1964 Helsinki declaration and its later amendments or comparable ethical standards. For this type of study, formal consent is not required.

\section{Study Population}

Treated aneurysms, which were evaluated with TOF-MRA, CE-MRA and CTP within two weeks after the operation, were taken into consideration. CT angiography views were created from CT perfusion views by the subtraction method.

Fifteen patients with saccular aneurysms were enrolled into the retrospective study from April 2013 to November 2016. One patient had two treated aneurysms. A total of 16 treated aneurysms were taken into consideration. These cases consisted of 11 females and 4 males. Their ages ranged from 25 years to 82 years with a mean of 52.8 years. Among them, 7 had hypertension and 2 had both hypertension and diabetes mellitus as co-morbidities. The location of the aneurysms and their treatment modalities are shown in Table I. Of the 16 aneurysms, 3 were coiled $\left(2 \times\right.$ Neuroform $^{\mathrm{TM}}$, Boston Scientific, Natick, MA; 1 x Driver ${ }^{\mathrm{TM}}$, Medtronic Inc., Minneapolis, MN). The other 13 aneurysms were surgically clipped (Sugita Titanium Aneurysm Clip. (Mizuho Ika Kogyo Co., Tokyo, Japan) (Table I).

\section{Radiological Imaging}

MRI was performed on a 1.5 Tesla system (Avanto; Siemens Medical Solution, Erlangen, Germany) with an 18-channel body coil and high performance gradients (maximum gradient, $45 \mathrm{mT} / \mathrm{m}$; maximum slew rate, $200 \mathrm{~T} / \mathrm{m} / \mathrm{s}$ ). All examinations were performed with a standard head coil.

\section{TOF MR Angiography}

3D-TOF images were acquired in the transverse plane with the following parameters: TR/ TE, 25/7 ms; flip angle, 25 degrees; 
Dundar TT. et al: Evaluation of Treated Intracranial Aneurysms

Table I: Location of Aneurysms and Their Treatment Modalities

\begin{tabular}{|c|c|c|}
\hline $\begin{array}{l}\text { Aneurysm } \\
\text { No }\end{array}$ & $\begin{array}{l}\text { Location of } \\
\text { Aneurysm }\end{array}$ & $\begin{array}{l}\text { Clipped/ } \\
\text { Coiled }\end{array}$ \\
\hline 2443099 & Supraclinoid ICA & Clipped \\
\hline 571805 & Supraclinoid ICA & Clipped \\
\hline 1537755 & Carotid Terminus & Clipped \\
\hline 291548 & Carotid Terminus & Coiled \\
\hline 2322412 & MCA trifurcation & Clipped \\
\hline 206481 & MCA trifurcation & Clipped \\
\hline 2470446 & M1 Saccular & Clipped \\
\hline 2390449 & Anterior communicating artery & Clipped \\
\hline 2390449 & Supraclinoid ICA & Clipped \\
\hline 2376613 & Anterior communicating Artery & Coiled \\
\hline 1152900 & Supraclinoid ICA & Clipped \\
\hline 2316786 & Supraclinoid ICA & Coiled \\
\hline 168303 & Anterior Communicating Artery & Clipped \\
\hline 1576940 & MCA trifurcation & Clipped \\
\hline 99555 & MCA trifurcation & Clipped \\
\hline 2264928 & MCA trifurcation & Clipped \\
\hline
\end{tabular}

ICA: Internal carotid artery, MCA: Middle cerebral artery, M1: Part of the middle cerebral artery from the origin to bifurcation/trifurcation.

matrix, $211 \times 256$; field of view (FOV), $200 \times 170 \mathrm{~mm}$; slice thickness, $0.7 \mathrm{~mm}$. Source images were then reconstructed using a maximum intensity projection (MIP) algorithm. Multiple projections using a large FOV were obtained every 15 degrees over 180 in lateral and anteroposterior views, providing 12 views in total.

\section{Contrast-Enhanced MR Angiography}

A CE-MRA fast imaging with steady-state precession (FISP) sequence with a rectilinear $\mathrm{k}$-space sampling (flip angle, 30; FOV, $230 \mathrm{~mm}$; matrix, 259×384; TR, 3.44; TE, 1.39; slice thickness, $1.0 \mathrm{~mm}$ ) was acquired in the coronal plane with an acquisition time of 40 seconds. A bolus of $0.2 \mathrm{mmol} /$ $\mathrm{kg}$ of gadolinium chelate (gadoterate meglumine; Dotarem, Guerbet, France) was injected at a rate of $2 \mathrm{~mL} / \mathrm{s}$ using an MRI-compatible power injector. Source images were then reconstructed using a MIP algorithm. Multiple projections using a large FOV were obtained every $15^{\circ}$ over 180 in lateral and anteroposterior views, providing 12 views in total.

\section{Volume CTP-CTA}

CTP was performed on a 128-row CT scanner (SOMATOM Definition, Flash; Siemens Healthcare, Erlangen, Germany). CTP covered the whole brain. The CTA data set was recovered from whole brain coverage CTP.
Volumetric CTP was performed in an adaptive 4D spiral mode (tube voltage, $80 \mathrm{kV}$; tube current-time product, 180 $\mathrm{mAs}$; collimation, $128 \times 0.6 \mathrm{~mm}$; rotation time, 0.3 seconds; CT dose index volume, $74 \mathrm{mGy}$ ). The coverage in the z-axis included $13 \mathrm{~cm}$ from the skull base. Thirty spiral scans were performed within 45 seconds. In the adaptive 4D spiral mode, the table continuously performs a smooth, periodic motion between 2 end positions while acquiring spiral scans. This generates multiple 3D scans that are sequential over time. The effective dose was $2.29 \mathrm{mSv}$. $45 \mathrm{~mL}$ of iodinated contrast iohexol (Omnipaque, $300 \mathrm{mg} / \mathrm{mL}$ of iodine; Amersham Health, Princeton, NJ) followed by $50 \mathrm{~mL}$ of saline flush, injected via an 18-gauge cannula into a cubital vein at a rate of $4.7 \mathrm{~mL} / \mathrm{s}$ using a power injector (Ulrich, Misouri). The Volumetric CT scan was initiated after a start delay of 4 seconds; 30 consecutive 3D datasets were reconstructed with the parameters described above. The time point with the highest opacification in vessels was selected from the dataset. These images were subtracted from first images in which there is no contrast material in the vessel. With subtraction, bone and artifact were removed. CTA data and their MIP reconstructions were created using the CT scanner workstation (Syngo; Siemens).

\section{Data Analysis}

Clipped or coiled aneurysms were evaluated by the 3 imaging techniques in terms of residue aneurysm and nearby vessel visualization. Perfusion abnormality was evaluated by CTP.

Scoring was used for visualization of vessels in the vicinity of aneurysms among the 3 imaging techniques. The best technique in depicting vessels nearby an aneurysm was scored as three points; the second best was scored as two points and the third one as one point. The highest total score belonged to the best imaging technique.

In the evaluation of residual aneurysms, the 3 imaging techniques were used to show the presence or absence of residual aneurysms. DSA was further performed in the presence of residual aneurysms.

\section{Statistical Analysis}

Measurements were entered into SPSS (ver. 19) and statistical analyses including Fisher's Exact Test and Cochran's $Q$ were carried out. Among the imaging techniques, the success of each imaging technique in the demonstration of residue aneurysm and nearby vessels was compared by Fisher's Exact Test. In each imaging technique, the differences among the three imaging techniques were evaluated by Cochran's $Q$ test. A significance level was set to 0.05 or lower.

\section{RESULTS}

A perfusion abnormality was revealed only by CTP. Following treatment by either coiling or clipping, a perfusion deficit with hypoperfusion, was present in $57 \%$ and hyperperfusion in $7 \%$ of the patients. The remaining $35 \%$ of the patients showed normal perfusion. Perfusion abnormality was much more common in clipped patients, of whom $72 \%$ showed hypoperfusion and 9\% hyperperfusion while the rest (18\%) of the clipped patients and all coiled patients showed normal perfusion. 
Among the three imaging techniques, vessel visualization in the vicinity of the aneurysm was best in sub-CTA, followed by CE-MRA and TOF-MRA, respectively.

In patients with clipped aneurysms, sub-CTA revealed residue aneurysms in $16.7 \%$ of the patients while TOF-MRA and CEMRA revealed none in the same population (Figure 1A-F). In patients with coiled aneurysms, CE-MRA revealed residue aneurysms in $100 \%$ and TOF-MRA in $33.3 \%$ while sub-CTA revealed none in the same population (Figures $2 A-E, 3 A-F$ ). Subsequently, residue aneurysms were all confirmed by DSA.

Although dramatic differences were noted in the evaluation of residue aneurysm as well as nearby vessel visualization, we could not find any statistical significance for either of them due to very few patients in the subcategories.

\section{DISCUSSION}

In the assessment of treated aneurysms, the imaging technique to be chosen should be non-invasive, easy and repeatable. Until recently, the gold standard technique has been catheter-based DSA that is usually performed at either 3 to 6 or 12 to 15 months depending on the institution's policy. However, DSA is invasive and carries a measurable degree of morbidity. Moreover, the technique of DSA includes patient discomfort, cost, and radiation exposure (17). In the literature, timing for evaluation of residue aneurysm by DSA is not clear, repeatability of DSA is not easy, and new non-invasive imaging techniques challenge the need for DSA. Therefore, DSA is not routinely used in the follow-up assessment of treated aneurysms at several institutes (1).

Recent studies have suggested non-invasive imaging techniques such as CT or MRI modalities $(24,26,27)$. Lately, the use of these modalities, such as CTA, 3D-TOF MRA and CE-MRA have increased but artifacts resulting from clip and coil cause a problem in determining the exact occlusion rates in treated aneurysms. High-attenuation platinum alloy coils causes marked beam hardening and streak artifact on CT and CTA, which typically obscure not only the aneurysm but also the adjacent parent and branch vessels as well as the surrounding brain parenchyma. Platinum alloy coils create relatively little distortion of the local magnetic field and, therefore, cause much less dramatic disruption of the MRI and MRA signal intensity $(3,22,23,25,26)$.
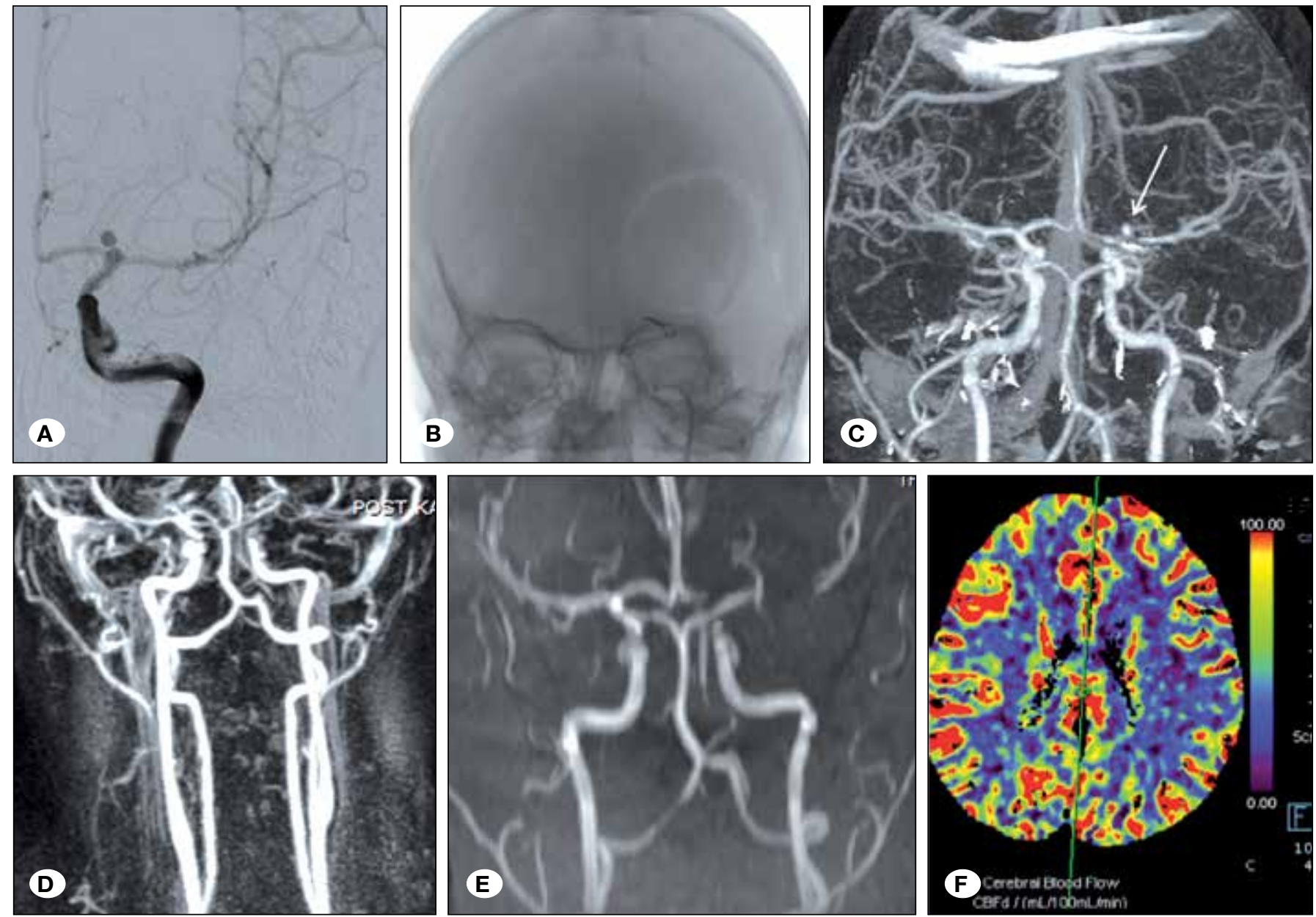

Figure 1: Left carotid terminus aneurysm (A) was clipped (B). On follow-up, C) sub-CTA reveals residual filling (arrow) that is not shown on CE-MRA (D) or 3D-TOF-MRA (E). Visualization of nearby vessel is best on sub-CTA in this case. Additionally, cerebral blood flow is decreased on left middle cerebral artery territory on CTP (F). 
In follow-up of intracranial aneurysms treated with endovascular coil occlusion, two different MRA techniques (3D-TOFMRA and CE-MRA) are discussed (18). Most have reported the superiority of CE-MRA $(3,12)$. CE-MRA has been shown to be as accurate as conventional catheter-based cerebral angiography. CE-MRA has replaced DSA for this purpose in many institutions. Several studies have compared CE-MRA to DSA and have shown that CE-MRA is an accurate technique to reveal aneurysmal remnants and re-canalization after endovascular coiling $(12,17,21)$. On the other hand, some studies also showed lower sensitivities of CE-MRA compared with the TOF-MRA for the detection of residual neck or residual aneurysm. Furthermore, TOF-MRA provides better spatial resolution than CE-MRA that is inherently not susceptible to saturation effects $(6,11,21)$. Some could not find any statistical difference between accuracies of 3D-TOF-MRA and CE-MRA with no added benefit of contrast administration $(5,8,12,20)$. Moreover some show that CE-MRA and 3D-TOF-MRA were less accurate than DSA in the detection of aneurysm remnant (12). Some reported that MRA is better than DSA because of artifacts resulting from subtraction methods and opaque coil packages limiting DSA. Finally, MRA has begun to replace DSA for the detection of residual or recurrent aneurysms in follow-up of aneurysms treated with endovascular coil occlu- sion (7), although some studies were still suspicious for MRA $(11,20)$. Furthermore, endovascular coil mass has high hyperdense artifact on CT techniques. These coils are designed to allow optimum visibility during endovascular treatments performed under fluoroscopic control. The coil-related artifacts typically obscure not only the aneurysm but also the adjacent parenchyma and branch vessels as well as the surrounding brain parenchyma on CT, CTA imaging. CT techniques also have radiation exposure hazards (7).

Technical advancements have made MRI techniques more effective. Several studies have reported 3T to be superior to 1.5 T when utilized for follow-up of coiled intracranial aneurysms, and although artifacts are more pronounced in 3T than 1.5T, remnant aneurysm are better shown in $3 \mathrm{~T}(7,15,22,23)$. MRI techniques, such as short TE acquisition and doubled receiver bandwidth, are used to decrease coil-related artifact in both CE-MRA and TOF-MRA $(21,22)$. CE-MRA carries a risk of nephrogenic fibrosis due to gadolinium -containing contrast agent in patients with chronic renal diseases (17).

In the follow-up of intracranial aneurysms treated with clipping, CE-MRA and TOF-MRA have been of limited value due to susceptibility artifacts of clips obscuring the region of interest. Some studies have shown that CE-MRA and TOF-MRA
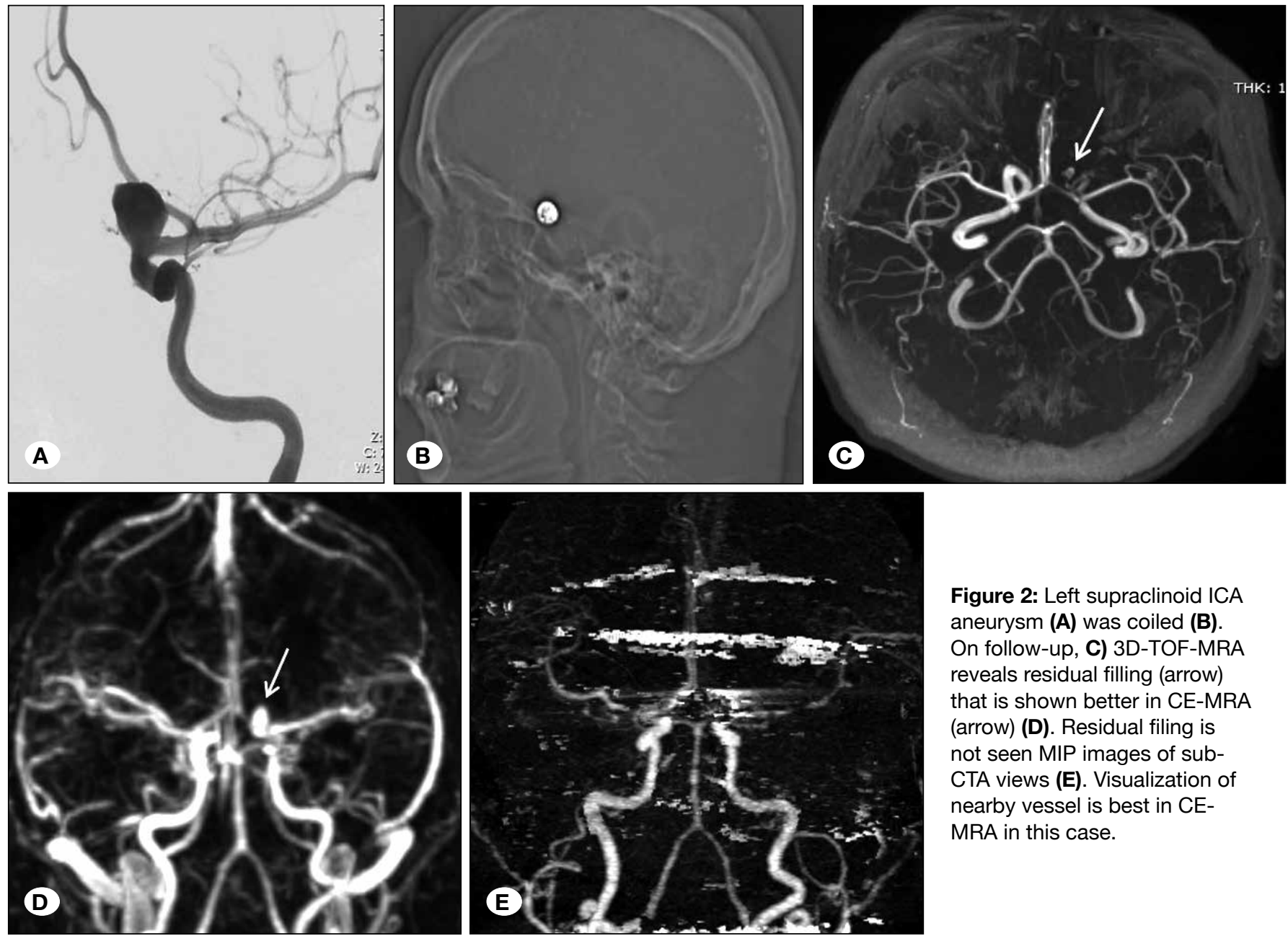

Figure 2: Left supraclinoid ICA aneurysm (A) was coiled (B). On follow-up, C) 3D-TOF-MRA reveals residual filling (arrow) that is shown better in CE-MRA (arrow) (D). Residual filing is not seen MIP images of subCTA views (E). Visualization of nearby vessel is best in CEMRA in this case. 

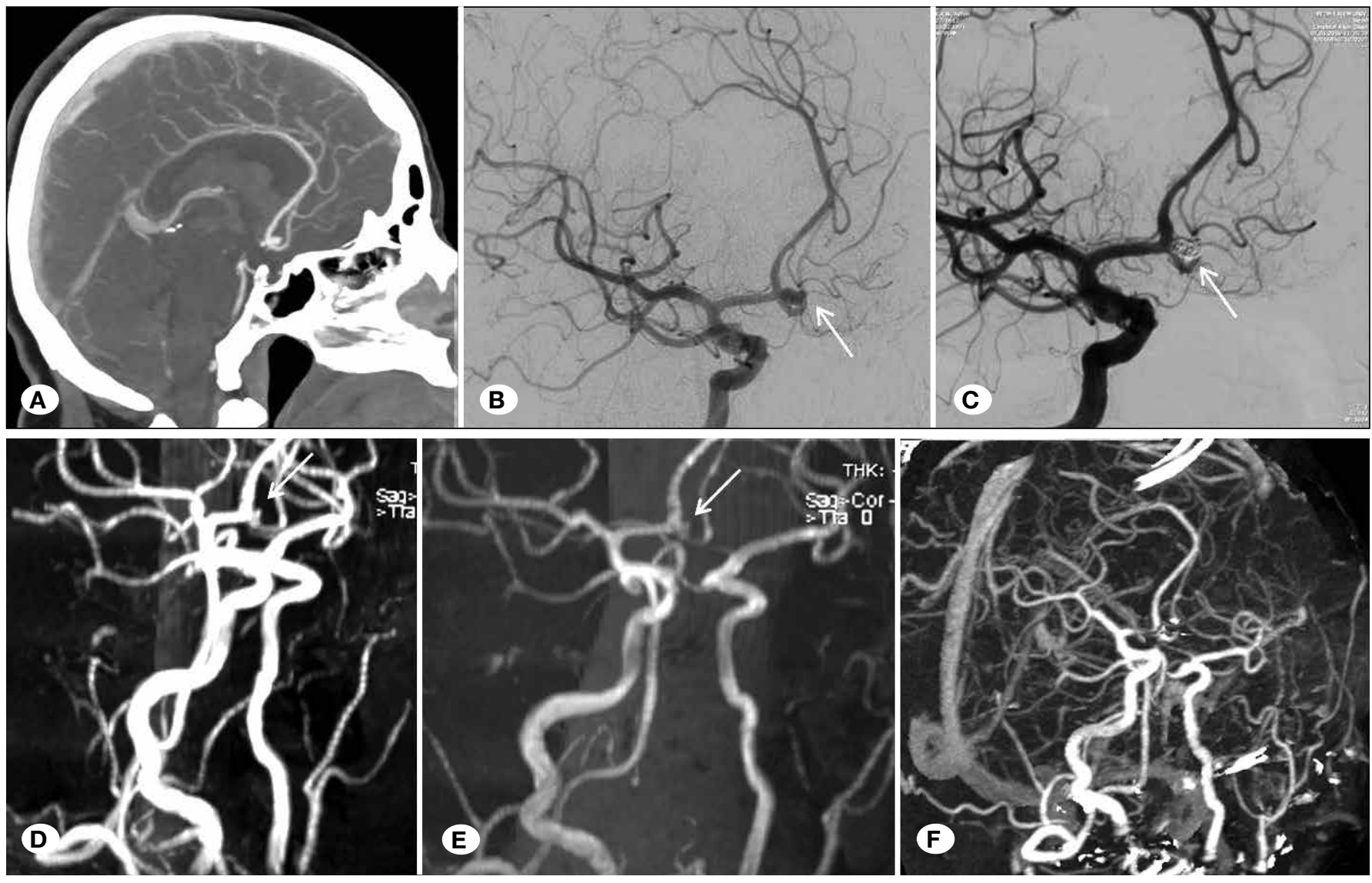

Figure 3: Anterior communicating artery aneurysm on CTA (A) was coiled with pre- (arrow on B) and postcoiling DSA views (arrow on C). On follow-up, MIP image of CE-MRA (arrow on D) and 3D-TOF-MRA (arrow on C) reveals residual filling that is not seen on MIP view of sub-CTA (F). Visualization of nearby vessel is best in CE-MRA in this case.

are of use in assessment of patients in whom artifacts were reduced by using titanium alloy clips $(6,9)$. Imaging after surgical aneurysm clipping has traditionally been evaluated with DSA. CTA may provide an acceptable alternative in many cases, particularly for long-term postoperative follow-up $(16,19,27)$. Recent studies reporting the role of CTA for postoperative evaluation of aneurysmal occlusion rates after surgical clipping suggest that CTA might be considered valuable in patients treated with titanium clips $(19,27,28)$. CTA was reported to have a higher sensitivity and specificity in the detection of residue-recurrent aneurysm, when compared to DSA, and especially residue-recurrent aneurysm larger than $2 \mathrm{~mm}$ (19). Artifacts from clips on CTA can also be reduced by using titanium alloy clips instead of cobalt alloy clips to detect small residual-recurrent aneurysms (1).

CTP is also a non-invasive technique. Nevertheless, residue aneurysm evaluation by CTA and CTP is not commonly used in clinical practice. CTP quantitatively evaluates cerebral hemodynamics to identify regions of potential ischemia due to vasospasm $(1,15)$. In our study, we evaluated remnant aneurysm with subtracted CTA from CTP. During CTP, consecutive images were taken as contrast agent went through the brain. If we subtracted the image with contrast in arterial vessels from the image with no contrast in the brain yet, only the arterial vessel can be seen. By subtraction, artifacts resulting from the clip can be erased. Our study is first study using sub-CTA from CTP in remnant aneurysm evaluation in clipped patients. We noticed that residue aneurysms of clipped patients being well depicted on sub-CTA comparison to the CE-MRA and TOF-MRA.

In the literature, many studies have compared MRA techniques with each other or MRA and DSA or DSA and CTA (4). Our study is first study comparing all three non-invasive imaging techniques (CE-MRA, TOF-MRA and sub-CTA from CTP) in the same population as well as sub-CTA from CTP, a new technique that has never been studied in the follow-up of treated aneurysms so far. We suggest that the sub-CTA form CTP should be used in the follow-up of clipped patients due to its success in artifact removal and demonstration of residue aneurysm.

Our study has some limitations. First, the few patients in subcategories hinder the statistical analysis although dramatic differences are present in the evaluation of residue aneurysm as well as nearby vessel visualization in subcategories. Second is we compared three imaging modalities with each other. We could not use DSA as a gold standard for all cases. 
Dundar TT. et al: Evaluation of Treated Intracranial Aneurysms

However the cases with residual aneurysm were all confirmed by DSA. Third, based on the limitations mentioned above, our results were suggestive not conclusive.

\section{CONCLUSION}

In the follow-up of treated aneurysms, the choice of imaging technique to be used should be made on both a patient-bypatient and clinical experience basis. However, we noticed that residual aneurysms were more commonly revealed by CE-MRA in coiled patients and they were more commonly and better shown in sub-CTA from CT perfusion in clipped patients. In addition, perfusion abnormality is more common in clipped patients compared to coiled ones. These non-invasive techniques can reduce or eliminate the need for conventional catheter-based angiography. Further studies with larger series comparing the effectiveness of CE- MRA, 3D-TOF-MRA and sub-CTA from CT perfusion in the evaluation of residual aneurysm are needed to enhance our results.

\section{REFERENCES}

1. Akyuz M, Tuncer R, Yilmaz S, Sindel T: Angiographic followup after surgical treatment of intracranial aneurysms. Acta Neurochir (Wien) 146:245-250, 2004

2. Aralasmak A, Akyuz M, Ozkaynak C, Sindel T, Tuncer R: CT angiography and perfusion imaging in patients with subarachnoid hemorrhage: Correlation of vasospasm to perfusion abnormality. Neuroradiology 51:85-93, 2009

3. Attali J, Benaissa A, Soize S, Kadziolka K, Portefaix C, Pierot L: Follow-up of intracranial aneurysms treated by flow diverter: Comparison of three-dimensional time-of-flight MR angiography (3D-TOF-MRA) and contrast-enhanced MR angiography (CE-MRA) sequences with digital subtraction angiography as the gold standard. J Neurointerv Surg 8:8186, 2016

4. Buhk, JH, Kallenberg, K, Mohr, A. Dechent P, Knauth M: Evaluation of angiographic computed tomography in the follow-up after endovascular treatment of cerebral aneurysms-A comparative study with DSA and TOF-MRA. Eur Radiol 19: 430-436, 2009

5. Cremers $\mathrm{CH}$, Van der Bilt IA, Van der Schaaf IC, Vergouwen MD, Dankbaar JW, Cramer MJ, Wilde AA, Rinkel GJ, Velthuis BK: Relationship between cardiac dysfunction and cerebral perfusion in patients with aneurysmal subarachnoid hemorrhage. Neurocrit Care 24:202-206, 2016

6. Gibbs GF, Huston J 3rd, Bernstein MA, Riederer SJ, Brown RD Jr: 3.0-Tesla MR angiography of intracranial aneurysms: Comparison of time-of-flight and contrast-enhanced techniques. J Magn Reson Imaging 21(2):97-102, 2005

7. Gramsch C, Zülow S, Nensa F, Maderwald S, Göricke S, Ringelstein A, Müller O, Sure U, Wanke I, Schlamann M: Can we now dispense with DSA in the evaluation of aneurysm occlusion even in the most crucial first follow-up after endovascular treatment? Clin Neurol Neurosurg 149:136-142, 2016
8. Guyatt GH, Oxman AD, Sultan S, Glasziou P, Akl EA, AlonsoCoello P, Atkins D, Kunz R, Brozek J, Montori V, Jaeschke R, Rind D, Dahm P, Meerpohl J, Vist G, Berliner E, Norris S, FalckYtter Y, Murad MH, Schünemann HJ; GRADE Working Group: GRADE guidelines, 9: Rating up the quality of evidence. J Clin Epidemiol 64:1311-1316, 2011

9. Hattingen E, Blasel S, Du Mesnil R, Vatter H, Friedhelm E, Zanella FE, Weidauer S: MR angiography in patients with subarachnoid hemorrhage: Adequate to evaluate vasospasminduced vascular narrowing? Neurosurg Rev 33:431-439, 2010

10. Kang HS, Han MH, Kwon BJ, Jung SI, Oh CW, Han DH, Chang $\mathrm{KH}$ : Postoperative 3D angiography in intracranial aneurysms. AJNR Am J Neuroradiol 25:1463-1469, 2004

11. Kwee TC, Kwee RM: MR angiography in the follow-up of intracranial aneurysms treated with Guglielmi detachable coils: Systematic review and meta-analysis. Neuroradiology 49:703-713, 2007

12. Marciano D, Soize S, Metaxas G, Portefaix C, Pierot L: Followup of intracranial aneurysms treated with stent-assisted coiling: Comparison of contrast-enhanced MRA, time-offlight MRA, and digital subtraction angiography. J Neuroradiol 44(1):44-51, 2017

13. Molyneux AJ, Kerr RS, Birks J, Ramzi N, Yarnold J, Sneade MM, Rischmiller J, ISAT Collaborators: Risk of recurrent subarachnoid haemorrhage, death, or dependence and standardised mortality ratios after clipping or coiling of an intracranial aneurysm in the International Subarachnoid Aneurysm Trial (ISAT): Long-term follow-up. Lancet Neuro 8: 427-433, 2009

14. Pierot L, Portefaix C, Boulin A, Gauvrit JY: Follow-up of coiled intracranial aneurysms: Comparison of $3 d$ time-of-flight and contrast-enhanced magnetic resonance angiography at $3 \mathrm{~T}$ in a large, prospective series. Eur Radiol 22:2255-2263, 2012

15. Sanelli PC, Ugorec I, Johnson CE, Tan J, Segal AZ, Fink M, Heier LA, Tsiouris AJ, Comunale JP, John M: Using quantitative $\mathrm{CT}$ perfusion for evaluation of delayed cerebral ischemia following aneurysmal subarachnoid hemorrhage. AJNR Am J Neuroradiol 32:2047-2053, 2011

16. Schunemann HJ, Oxman AD, Brozek J, Glasziou P, Jaeschke R, Vist GE, Williams JW, Kunz R, Craig J, Montori VM, Bossuyt P, Guyatt GH: Grading quality of evidence and strength of recommendations for diagnostic tests and strategies. BMJ 336:1106 -1110, 2008

17. Shankar JJ, Lum C, Parikh N, dos Santos M: Long-term prospective follow-up of intracranial aneurysms treated with endovascular coiling using contrast-enhanced MR angiography. AJNR Am J Neuroradiol 31:1211-1215, 2010

18. Spetzler RF, McDougall CG, Zabramski JM, Albuquerque FC, Hills NK, Russin JJ, Partovi S, Nakaji P, Wallace RC: The Barrow Ruptured Aneurysm Trial: 6-year results. J Neurosurg 123:609-617, 2015

19. Uysal E, Ozel A, Erturk SM, Kirdar O, Basak M: Comparison of multislice computed tomography angiography and digital subtraction angiography in the detection of residual or recurrent aneurysm after surgical clipping with titanium clips. Acta Neurochir (Wien) 151:131-135, 2009 
20. Van Amerongen MJ, Boogaarts HD, De Vries J, Verbeek AL, Meijer FJ, Prokop M, Bartels RH: MRA versus DSA for followup of coiled intracranial aneurysms: A meta-analysis. AJNR Am J Neuroradiol 35:1655- 1661, 2014

21. Van Rooij WJ, Sluzewski M: Opinion: Imaging follow-up after coiling of intracranial aneurysms. AJNR 30:1646-1648, 2009

22. Wallace RC, Karis JP, Partovi S, Fiorella D: Noninvasive imaging of treated cerebral aneurysms, Part I: MR angiographic followup of coiled aneurysms. AJNR Am J Neuroradiol 27:10011008, 2007

23. Wallace RC, Karis JP, Partovi S, Fiorella D: Noninvasive imaging of treated cerebral aneurysms, Part II: CT angiographic followup of surgically clipped aneurysms. AJNR Am J Neuroradiol 28:1207-1212, 2007

24. Weber W, Yousry TA, Felber SR, Henkes H, Nahser HC, Roar N, Kühne D: Noninvasive follow-up of GDC-treated saccular aneurysms by MR angiography. Eur Radiol 11:1792-1797, 2001
25. White PM, Teasdale EM, Wardlaw JM, Easton V: Intracranial aneurysms: CT angiography and MR angiography for detection prospective blinded comparison in a large patient cohort. Radiology 219:739-749, 2001

26. Willinsky RA, Taylor SM, TerBrugge K, Farb RI, Tomlinson G, Montanera W: Neurologic complications of cerebral angiography: Prospective analysis of 2,899 procedures and review of the literature. Radiology 227:522-528, 2003

27. Yoon NK, McNally S, Taussky P, Park MS: Imaging of cerebral aneurysms: A clinical perspective. Neurovascular Imaging 2: 6, 2016

28. Zachenhofer I, Cejna M, Schuster A, Donat M, Roessler K: Image quality and artefact generation post-cerebral aneurysm clipping using a 64-row multislice computer tomography angiography (MSCTA) technology: A retrospective study and review of the literature. Clin Neurol Neurosurg 112:386-391, 2010 